\title{
Cystic Lesions of the Pancreas: Observe or Operate
}

\author{
Peter J. Allen ${ }^{1,2}$
}

Received: 24 September 2015 / Accepted: 30 September 2015 / Published online: 8 October 2015

(C) Association of Surgeons of India 2015

A cystic lesion of the pancreas is a radiographic finding that has a broad histologic differential. This differential includes non-neoplastic pseudocysts, benign neoplastic cysts, premalignant cysts, and cystic lesions with invasive carcinoma [1]. Because of the increased use of high-quality cross-sectional imaging, an increasing number of patients are being identified with small asymptomatic cysts, and the management of these patients has become very controversial $[2,3]$. This controversy has centered on the inability to determine the exact histopathologic diagnosis without resection. Because of this diagnostic uncertainty, many authors have recommended routine resection of all pancreatic cysts $[4,5]$. It should be emphasized, however, that the ability to determine the histologic diagnosis of pancreatic cysts without resection continues to improve, and current imaging and endoscopic techniques can typically differentiate benign serous from pre-cancerous mucinous cysts of the pancreas. In addition, even within the mucinous sub-group, our ability to detect high-risk lesions is improving.

Because of these improvements in diagnosis, we have recommended a more selective approach to resection [6-8]. We have found that with current imaging techniques, and with an improved understanding of the various histologic entities, a group of patients can be identified who have an extremely low risk of malignancy. Within this group of patients, we routinely recommend non-operative management (radiographic follow-

Peter J. Allen

allenp@mskcc.org

1 HPB Services, Memorial Sloan Kettering Cancer Center, New York, NY, USA

2 Rubenstein Center for Pancreatic Cancer Research, Memorial Sloan Kettering Cancer Center, New York, NY 10021, USA up). These patients typically have small $(<3 \mathrm{~cm})$, incidentally discovered cysts of the pancreas that do not have a solid component or other concerning clinical or radiographic features of malignancy (such as main pancreatic duct dilation). This selective approach avoids the risks of operation in patients with benign lesions, but with current limitations in non-resectional diagnosis, cannot guarantee that a malignancy is not mistakenly being observed. Operative mortality rates following pancreatectomy range between 2 and $15 \%$ and significant postoperative complications occur in approximately $45 \%$ of patients [9]. Our data suggest that with current imaging and endoscopic techniques, a group of patients with pancreatic cysts can be identified with a malignancy rate of $<3 \%$ [8]. In this group of patients, the risk of death from pancreatectomy exceeds the risk of the lesion being malignant and radiographic surveillance should be recommended.

When the histopathology of a given cyst can be determined non-operatively, the treatment recommendations may be less challenging. In these instances, treatment recommendations can be made based on the known natural history of the specific histologic entity. For instance, when diagnostic evaluation identifies a patient with a serous cystadenoma (SCA), radiographic surveillance should be the routine recommendation. Resection for SCA should be reserved for the symptomatic patient, or in a healthy patient in whom significant growth has been observed. Patients identified with mucinous cystic neoplasms (MCN) typically undergo resection. These precancerous tumors are generally resected as they are much more common in the tail of the pancreas and are often found in young women ( $<40$ years), and after resection, there does not appear to be a risk for recurrence within the pancreatic remnant.

Presently, the most challenging group of patients is those with intraductal papillary mucinous neoplasms (IPMN). Controversy over the management of these patients exists because 
of the difficulty in identifying those with high-grade dysplasia, the known predilection for whole gland involvement, and the lack of understanding as to the frequency and timing of malignant progression. Controversy also exists over the extent of operation.

Patient discussion regarding operative resection of IPMN should include the risks of the operation, the risks of malignancy of the specific lesion, and the future risk of developing malignancy within the remnant gland if partial pancreatectomy is pursued. When cross-sectional imaging and endoscopic studies are characteristic of main duct IPMN (MD-IPMN), then operative resection is typically recommended as the risk of high-grade dysplasia or invasive disease is as high as 40 $50 \%$. A larger controversy regarding management of MDIPMN is over the extent of pancreatic resection. Because many consider IPMN to represent a defect within the entire ductal system, there is concern that removal of just part of the pancreas is inadequate. Patients who undergo less than total pancreatectomy for non-invasive IPMN have been found to have the potential to develop recurrent disease in the pancreatic remnant. A recent study from our center of 79 patients who underwent resection for non-invasive IPMN identified invasive remnant gland recurrence in $8 \%$ of patients after a median follow-up of 36 months [10]. This is certainly a concern; however, given the relatively low rate of remnant recurrence, we do not at this time recommend total pancreatectomy for all patients with MD-IPMN. When there is diffuse gland involvement radiographically, and when there is no overt radiographic evidence of invasive disease, total pancreatectomy may be warranted in selected patients with MD-IPMN. When less than total pancreatectomy is performed, postoperative surveillance is necessary in all patients.

Because of the low risk of malignancy in small BD-IPMN (branch duct IPMN), and because of the risks associated with pancreatectomy, our current approach to resection of the incidentally discovered BD-IPMN is selective. Resection is generally performed in the setting of symptoms, or concerning radiographic features (solid component, increasing size). Our typical follow-up schedule for patients undergoing nonoperative management consists of high-quality cross-sectional imaging every 6 months for 2 years, and then annually thereafter. Resection is typically performed when there is any radiographic change in the lesion such as significant growth, the development of a solid component, or mural nodularity.

In summary, the number of patients being identified with asymptomatic small cysts $(<3 \mathrm{~cm})$ of the pancreas is increasing. The majority of these patients can be safely monitored without operative resection. Resection should be reserved for those patients with presumed pre-cancerous cysts where there is concern for the development of malignancy (size, solid component, main duct dilation). The current challenges are to improve the sensitivity and specificity for the identification of mucinous sub-type, to better characterize the progression of IPMN, and to develop better methods for identifying the presence of in situ or invasive disease in these patients.

Conflict of Interest None.

\section{References}

1. Kloppel G, Kosmahl M (2001) Cystic lesions and neoplasms of the pancreas. The features are becoming clearer. Pancreatology 1:648655

2. Gorin AD, Sackier JM (1997) Incidental detection of cystic neoplasms of the pancreas. Md Med J 46:79-82

3. Fernandez-Del CC, Targarona J, Thayer SP et al (2003) Incidental pancreatic cysts: clinicopathologic characteristics and comparison with symptomatic patients. Arch Surg 138:427-3

4. Horvath KD, Chabot JA (1999) An aggressive resectional approach to cystic neoplasms of the pancreas. Am J Surg 178:269-274

5. Spinelli KS, Fromwiller TE, Daniel RA et al (2004) Cystic pancreatic neoplasms: observe or operate. Ann Surg 239:651-657

6. Allen PJ, Jaques DP, D'Angelica M et al (2003) Cystic lesions of the pancreas: selection criteria for operative and nonoperative management in 209 patients. J Gastrointest Surg 7:970-977

7. Allen PJ, D'Angelica M, Gonen M et al (2006) A selective approach to the resection of cystic lesions of the pancreas: results from 539 consecutive patients. Ann Surg 244:572-577

8. Gaujoux S, Brennan MF, Gonen M et al (2011) Cystic lesions of the pancreas: changes in the presentation and management of 1,424 patients at a single institution over a 15 -year time period. J Am Coll Surg 212:590-600

9. Birkmeyer JD, Finlayson SR, Tosteson AN et al (1999) Effect of hospital volume on in-hospital mortality with pancreaticoduodenectomy. Surgery 125:250-256

10. White R, D'Angelica M, Katabi N et al (2007) Fate of the remnant pancreas after resection of noninvasive intraductal papillary mucinous neoplasm. J Am Coll Surg 204:987-993 